\title{
Transcriptional Control of Hepatic Lipid Metabolism by SREBP and ChREBP
}

\author{
$\mathrm{Xu} \mathrm{Xu}, \mathrm{PhD}^{1} \quad$ Jae-Seon So, $\mathrm{PhD}^{1} \quad$ Jong-Gil Park, $\mathrm{PhD}^{1} \quad$ Ann-Hwee Lee, $\mathrm{PhD}^{1}$
}

${ }^{1}$ Department of Pathology and Laboratory Medicine, Weill Cornell
Medical College, New York, New York

Semin Liver Dis 2013;33:301-311.

\begin{abstract}
Address for correspondence Ann-Hwee Lee, PhD, Department of Pathology and Laboratory Medicine, Weill Cornell Medical College, 1300 York Avenue, New York, NY 10065 (e-mail: anl2042@med.cornell.edu).
\end{abstract}

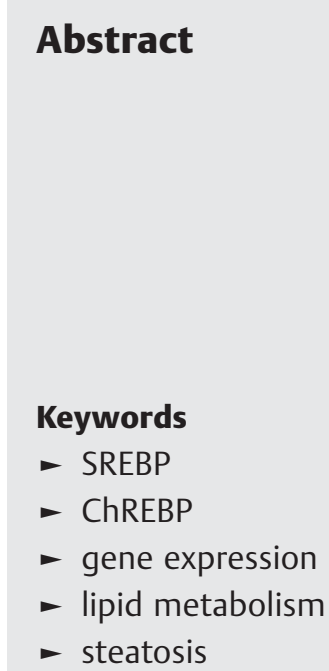

The liver is a central organ that controls systemic energy homeostasis and nutrient metabolism. Dietary carbohydrates and lipids, and fatty acids derived from adipose tissue are delivered to the liver, and utilized for gluconeogenesis, lipogenesis, and ketogenesis, which are tightly regulated by hormonal and neural signals. Hepatic lipogenesis is activated primarily by insulin that is secreted from the pancreas after a high-carbohydrate meal. Sterol regulatory element binding protein-1c (SREBP-1c) and carbohydrate-responsive element-binding protein (ChREBP) are major transcriptional regulators that induce key lipogenic enzymes to promote lipogenesis in the liver. Sterol regulatory element binding protein- $1 \mathrm{c}$ is activated by insulin through complex signaling cascades that control SREBP-1c at both transcriptional and posttranslational levels. Carbohydrate-responsive element-binding protein is activated by glucose independently of insulin. Here, the authors attempt to summarize the current understanding of the molecular mechanism for the transcriptional regulation of hepatic lipogenesis, focusing on recent studies that explore the signaling pathways controlling SREBPs and ChREBP.
Mammals adapt to the fluctuation of nutrient availability by storing surplus nutrient mainly in adipose tissue in the form of triglyceride (TG). Ingestion of carbohydrates stimulates the conversion of carbohydrate into TG in the liver, which is followed by the mobilization of TG from the liver to adipose tissue for long-term storage. Increased glucose levels in the circulation after a high-carbohydrate meal activate hepatic lipogenesis through multiple mechanisms. Pancreatic hormones, glucagon, and insulin play central roles in the regulation of both glucose and lipid metabolism. Glucose triggers insulin secretion from pancreatic $\beta$ cells, which stimulates glucose uptake and utilization, and promotes glycogen synthesis and lipogenesis in the liver. Insulin also suppresses hepatic glucose production, fat oxidation, and ketogenesis, shifting the balance to fat storage. Glucose itself also acts as a signaling molecule to regulate the genes encoding important enzymes in glycolysis and lipogenesis. ${ }^{1}$

Metabolic and hormonal cues such as glucose, insulin, and glucagon regulate the gene-expression program of glycolysis and lipogenesis via transcription factors. Sterol regulatory element binding protein-1c (SREBP-1c) is considered the master transcriptional regulator of fatty acid and TG synthesis in response to insulin stimulation. SREBP-1c is expressed at a low level in the liver of fasted animals, but is dramatically induced upon feeding, which is mediated by insulin. ${ }^{2,3}$ The function of SREBP-1c is also activated by insulin at the posttranslational level. Activated SREBP-1c binds to SRE (sterol regulatory element) sequences found on the promoters of its target genes as a homodimer. Sterol regulatory element binding protein-1c induces mRNAs encoding enzymes catalyzing various steps in the fatty acid and TG synthesis pathway, such as ATP-citrate lyase (ACL), acetylCoA synthetase (ACS), acetyl-CoA carboxylase (ACC), fatty acid synthase (FAS), stearoyl-CoA desaturase-1 (SCD1), and glycerol-3-phosphate acyltransferase (GPAT). ${ }^{2,4,5}$

Carbohydrate-responsive element-binding protein (ChREBP) has been recognized as a transcription factor that is activated by high glucose independent of insulin, and plays a key role in glycolysis and lipogenesis. ${ }^{1}$ It induces L-type pyruvate kinase (L-PK), ACC, and FAS genes by directly
Issue Theme Lipids and the Liver; Guest Editor, David E. Cohen, MD, PhD
Copyright (c) 2013 by Thieme Medical Publishers, Inc., 333 Seventh Avenue, New York, NY 10001, USA. Tel: +1(212) 584-4662.
DOI http://dx.doi.org/ 10.1055/s-0033-1358523. ISSN $0272-8087$. 


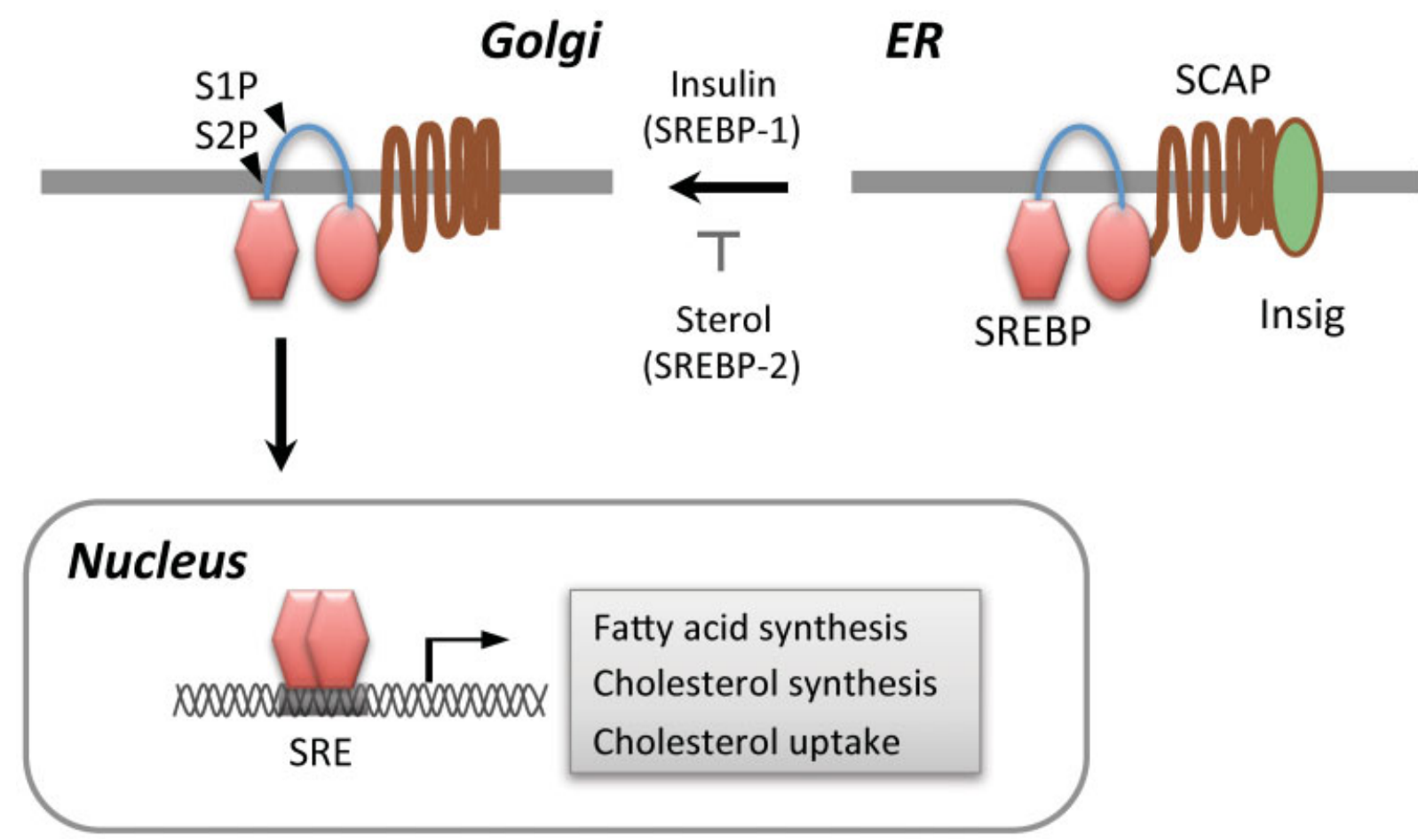

Fig. 1 Schematic illustration of the proteolytic activation of sterol regulatory element binding proteins (SREBPs). Sterol regulatory element binding proteins are synthesized as endoplasmic reticulum- (ER-) anchored precursor forms. Low cellular sterol concentrations trigger the release of the SREBP cleavage activating protein- (SCAP- SREBP-2 complex from Insig. Insulin stimulates the transport of SREBP-1C to the Golgi. Sterol regulatory element binding protein is sequentially cleaved by S1P and S2P proteases in the Golgi apparatus. The processed SREBP enters the nucleus to activate the transcription of genes regulating fatty acid and cholesterol metabolism.

binding to carbohydrate response elements (ChoRE) found in their promoters. ${ }^{6-8}$ Carbohydrate-responsive element-binding protein is a bZIP transcription factor that forms a heterodimeric complex with another bZIP protein, Max-like protein $\mathrm{X}(\mathrm{MLX}){ }^{9}$

During recent years, significant advancement has been made in our understanding of the mechanisms by which SREBP and ChREBP are activated in the liver and regulate lipid metabolism. In this review, we will focus on recent studies that provide new insights into the transcriptional regulation of hepatic lipid metabolism.

\section{SREBP Transcription Factors}

Sterol regulatory element binding proteins are major transcription factors that regulate the expression of genes involved in fatty acid, TG, and cholesterol metabolism in the liver. $^{10-12}$ The SREBP family consists of SREBP-1a, SREBP-1c, and SREBP-2. ${ }^{13,14}$ Both SREBP-1a and SREBP-1c are encoded by a single gene, but are transcribed by different promoters, producing similar proteins that differ only in the N-terminal region. ${ }^{14}$ Whereas SREBP-1c is the predominant isoform expressed in the liver, SREBP-1a is produced in certain cell types in the immune system as well as in cultured cell lines. ${ }^{14,15}$ Although there is some functional overlap between different isoforms, SREBP-1c is mostly responsible for the expression of genes involved in fatty acid biosynthesis, whereas SREBP-2 activates cholesterol metabolism genes. ${ }^{10}$
Sterol regulatory element binding proteins are synthesized as precursor forms containing two transmembrane helices that anchor the protein in the endoplasmic reticulum (ER) membrane (- Fig. 1). ${ }^{16}$ They are associated with the SREBP cleavage activating protein (SCAP) and ER retention protein called Insig. ${ }^{17}$ To be activated, the SREBP-SCAP complex should be dissociated from Insig, associate with COPII-coated vesicles, and then migrate to the Golgi apparatus. ${ }^{18,19}$ Sterol regulatory element binding proteins are sequentially cleaved by site 1 (S1P) and site 2 (S2P) proteases in the Golgi, which releases the N-terminal cytosolic portion of the protein that enters the nucleus to act as the active transcription factor. ${ }^{10}$

\section{Regulation of SREBP Activation by Proteolytic Cleavage}

SREBP cleavage activating protein is a polytopic protein containing eight transmembrane helices and seven loops. ${ }^{20}$ Transmembrane helices 2 to 6 are required for the binding of SCAP to Insig. ${ }^{18,21,22}$ Cholesterol binds to SCAP in loop 1 located in the ER lumen, which triggers a conformational change in loop 6 facing the cytoplasm. ${ }^{18,21,22}$ This conformational change of SCAP precludes its interaction with COPII proteins, and hence suppresses the mobilization of the SCAPSREBP-2 complex to the Golgi. When the ER cholesterol level is decreased, the SCAP-SREBP-2 complex binds to COPII vesicles to be transported to the Golgi for proteolytic 
activation. SCAP responds to the changes in ER cholesterol concentration with high precision, such that small changes in the ER cholesterol levels from the threshold level (5\%) abruptly turn on/off SCAP-SREBP-2 association with COPII and the consequent SPREP-2 activation, enabling the precise regulation of SREBP-2 by cholesterol abundance. ${ }^{21,22}$

It is well known that insulin transcriptionally activates SREBP-1c in the liver. ${ }^{3,23}$ But it has been less clear whether insulin also stimulates proteolytic processing of SREBP-1c because it is technically difficult to distinguish the contribution by the transcriptional activation and the proteolytic processing to the increased nuclear SREBP-1c level in response to insulin stimulation. To distinguish the effect of insulin on SREBP-1c processing from the transcriptional activation of SREBP1c mRNA, Hegarty et al pretreated rat hepatocytes with the LXR $\alpha$ agonist TO-901317 before adding insulin. ${ }^{24}$ TO-901317 induced SREBP-1c mRNA, which was not further increased by insulin. Under such conditions, insulin significantly increased the processed nuclear SREBP1c protein, indicating that insulin stimulated SREBP-1c processing. Similarly, insulin increased the nuclear processed SREBP-1c level in hepatocytes infected with SREBP-1c adenovirus or in transgenic rat liver that expressed human SREBP1c under control of the apoE promoter. ${ }^{25,26}$ In contrast, insulin did not increase SREBP-2 processing, highlighting the specific role of insulin in SREBP-1c processing. ${ }^{25}$ These independent studies clearly demonstrate that insulin not only activates SREBP-1c transcription, but also stimulates SREBP$1 c$ processing.

How does insulin stimulate SREBP-1c processing? Stimulation of SREBP-1c processing by insulin was inhibited by small molecule inhibitors of phosphoinositide 3-kinase (PI3K), Akt, and mammalian target of rapamycin (mTOR) complex 1 (mTORC1), and p70 ribosomal S6 kinase (p70S6K), indicating that the PI3K/mTOR signaling pathway plays a critical role in SREBP-1c processing. ${ }^{24-27}$ It has been proposed that Akt directly phosphorylates SREBP-1c, which increases the affinity of SCAP-SREBP-1c complex for Sar1/ Sec23/24 proteins of COPII-coated vesicles and facilitates the Golgi transportation of SREBP-1c. ${ }^{25}$ Interestingly, insulin strongly suppresses the expression of Insig-2a, which is the major Insig isoform expressed in the liver. ${ }^{28}$ The Insig2 gene has two different promoters, from which Insig-2a and Insig$2 \mathrm{~b}$ mRNAs are transcribed. These two transcripts differ in noncoding exon 1 , and hence produce same protein. Suppression of Insig-2a requires Akt activation, and involves mRNA destabilization. ${ }^{27,29}$ It is notable that Insig-2a preferentially interacts with the SCAP-SREBP-1c complex, while Insig-1 binds to SCAP-SREBP- $2 .{ }^{29}$ Hence, it is conceivable that insulin selectively activates SREBP-1c processing through two distinct mechanisms that involve the suppression of Insig-2a and the induction of SREBP-1c phosphorylation, which facilitates the association of SCAP-SREBP-1c complex with COPII-coated vesicles ( - Fig. 2). However, the precise mechanism by which insulin stimulates SREBP-1c processing remains to be further investigated. For example, although SREBP-1c activation correlates well inversely with the Insig-2a level, it is not known if the disappearance of Insig-2a protein precedes SREBP-1c
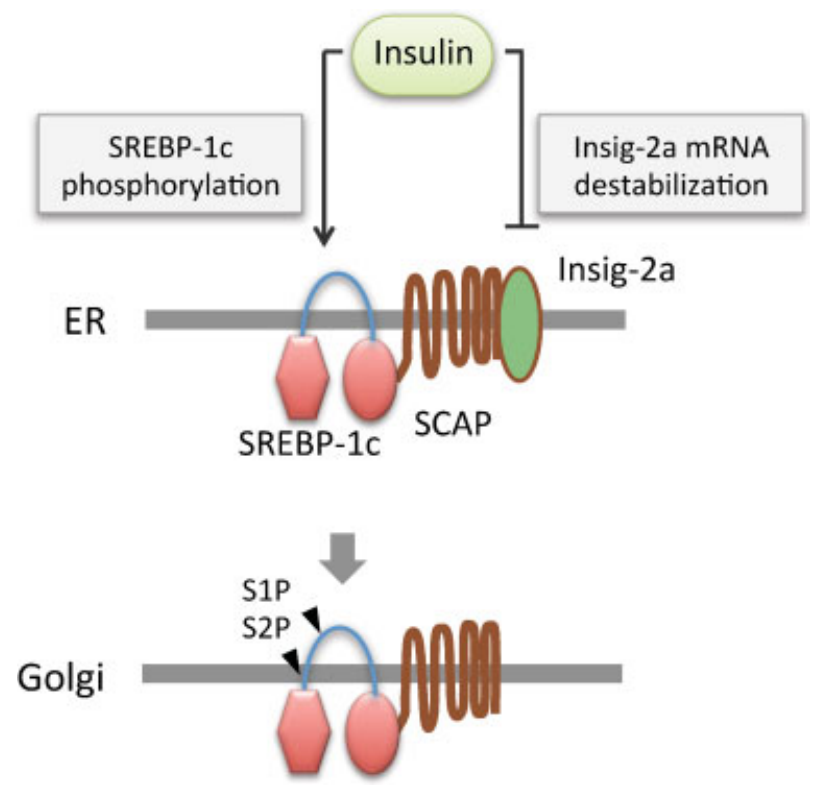

Fig. 2 Insulin promotes sterol regulatory element binding protein 1c (SREBP-1c) processing. Insulin induces AKT-mediated SREBP-1c phosphorylation, which stimulates the transport of SREBP-1c-SREBP cleavage activating protein (SCAP) complex to the Golgi apparatus. Insulin also induces the degradation of Insig-2a mRNA to promote the Golgi transport and proteolytic processing of SREBP-1c.

activation. Furthermore, Insig-2a is strongly induced by the LXR $\alpha$ agonist TO-901317, but does not suppress insulinmediated SREBP-1c processing, suggesting that Insig-2a downregulation is not a prerequisite for SREBP-1c processing. ${ }^{24}$ It is possible that the decreased expression of Insig-2a contributes to SREBP-1c processing upon chronic insulin stimulation, while acute insulin stimulation activates SREBP-1c processing through a distinct mechanism that does not involve Insig-2a downregulation.

\section{Regulation of SREBP Activation by Nuclear Translocation}

Although the processed SREBPs contain a nuclear localization signal in the HLH-Zip domain that mediates spontaneous import of the protein to the nucleus, ${ }^{30}$ a recent study suggests that the nuclear entry of processed SREBP-1 and SREBP-2 could be regulated by mTORC $1 .{ }^{31}$ Lipin 1 , a phosphatidic acid phosphatase, is an mTORC1 substrate. Dephosphorylation of lipin 1 by mTOR inhibitor treatment triggers the entry of lipin 1 into the nucleus. Interestingly, dephosphorylated nuclear lipin 1 inhibits nuclear localization of SPREBPs in NIH-3T3 cells. In the presence of lipin 1 in the nucleus, SREBPs appear to localize to the perinuclear area in proximity to the nuclear matrix component lamin A. A lipin 1 construct carrying a mutation in the MTOC1 phosphorylation site suppressed the nuclear entry of processed SREBP-1c and the expression of lipogenic target genes, indicating that lipin-1 suppresses the transcriptional function of SREBPs by suppressing their nuclear localization. Lipin 1 appears to be critically involved in SREBP regulation by mTORC1 in mouse liver as well because 
lipin 1 silencing restored lipogenic gene expression in the liver of Raptor knockout mice, where mTORC1 was inactivated. Lipin 1 also regulates fatty acid metabolism through other mechanisms. Lipin 1 dephosphorylates phosphatidic acid to produce diacylglycerol. ${ }^{32,33}$ It also stimulates fatty acid oxidation in concert with peroxisome proliferator-activated receptor $\alpha(P P A R \alpha)$ and its coactivators. ${ }^{34}$ Further studies will reveal the significance of lipin 1 regulation of SREBP in hepatic lipid metabolism under various pathophysiological conditions.

\section{Transcriptional Regulation of SREBP-1C}

Hepatic SREBP-1c mRNA level is dynamically regulated by nutritional status. SREBP-1c mRNA expression in liver is suppressed in fasted animals, and highly induced by ingestion of a high carbohydrate diet. ${ }^{2}$ Expression of SREBP-1c was suppressed in diabetic rats induced by streptozotocin treatment, but normalized by insulin injection, indicating that insulin mediates the induction of SREBP-1c mRNA by carbohydrate diet ingestion. ${ }^{23}$ Insulin strongly induces SREBP-1c mRNA in cultured hepatocytes. ${ }^{14,35}$ In contrast, glucagon suppresses SREBP-1c mRNA expression via the cyclic adenosine 3',5'-monophosphate/protein kinase A signaling pathway. ${ }^{35,36}$

The engagement of insulin with its cell surface receptor induces the phosphorylation of the scaffolding protein family insulin-receptor substrates (IRS), which then initiates a signaling cascade that culminates with the transcriptional suppression of gluconeogenesis and the activation of lipogenesis (-Fig. 3). ${ }^{37,38}$ Tyrosine phosphorylation of IRS by the insulin receptor recruits phosphoinositide-3-kinase (PI3K), which then phosphorylates phosphatidylinositol $(4,5)$ bisphosphate (PtdIns $\left.(4,5) \mathrm{P}_{2}\right)$ to produce $\operatorname{Ptd}(3,4,5) \mathrm{P}_{3}\left(\mathrm{PIP}_{3}\right.$. As a phospholipid second messenger, $\mathrm{PIP}_{3}$ recruits the Ser/Thr kinase AKT to the plasma membrane, where it is phosphorylated by 3-phosphoinositide-dependent protein kinase-1 (PDK1) to be activated. Consequently, active AKT phosphorylates a wide range of downstream targets involved in cell metabolism, such as forkhead box protein 01 (Foxo1), glycogen synthase kinase 3 (GSK3), and tuberous sclerosis 2 (TSC2) within the TSC1-TSC2 complex. ${ }^{39,40}$ Tuberous sclerosis 2 is a critical regulator of the mammalian TOR (MTOR) complex 1 (mTORC1), which plays a central role in cell growth and metabolism. ${ }^{41,42}$ Phosphorylation of TSC2 by AKT results in the activation of mTORC1, as the phosphorylated TSC2 no longer inhibits the Ras homolog enriched in brain (Rheb) protein that is critically required for mTORC1 functions. ${ }^{40}$

Recently, mTORC1 has emerged as an important regulator of SREBP-1c that activates both SREBP-1c transcription $26,43,44$ and the proteolytic processing in response to insulin stimulation. $^{24-27}$ Suppression of mTORC1 activity by rapamycin inhibited the expression of SREBP-1c and lipogenic genes regulated by SREBP-1c in the livers of rodents subjected to

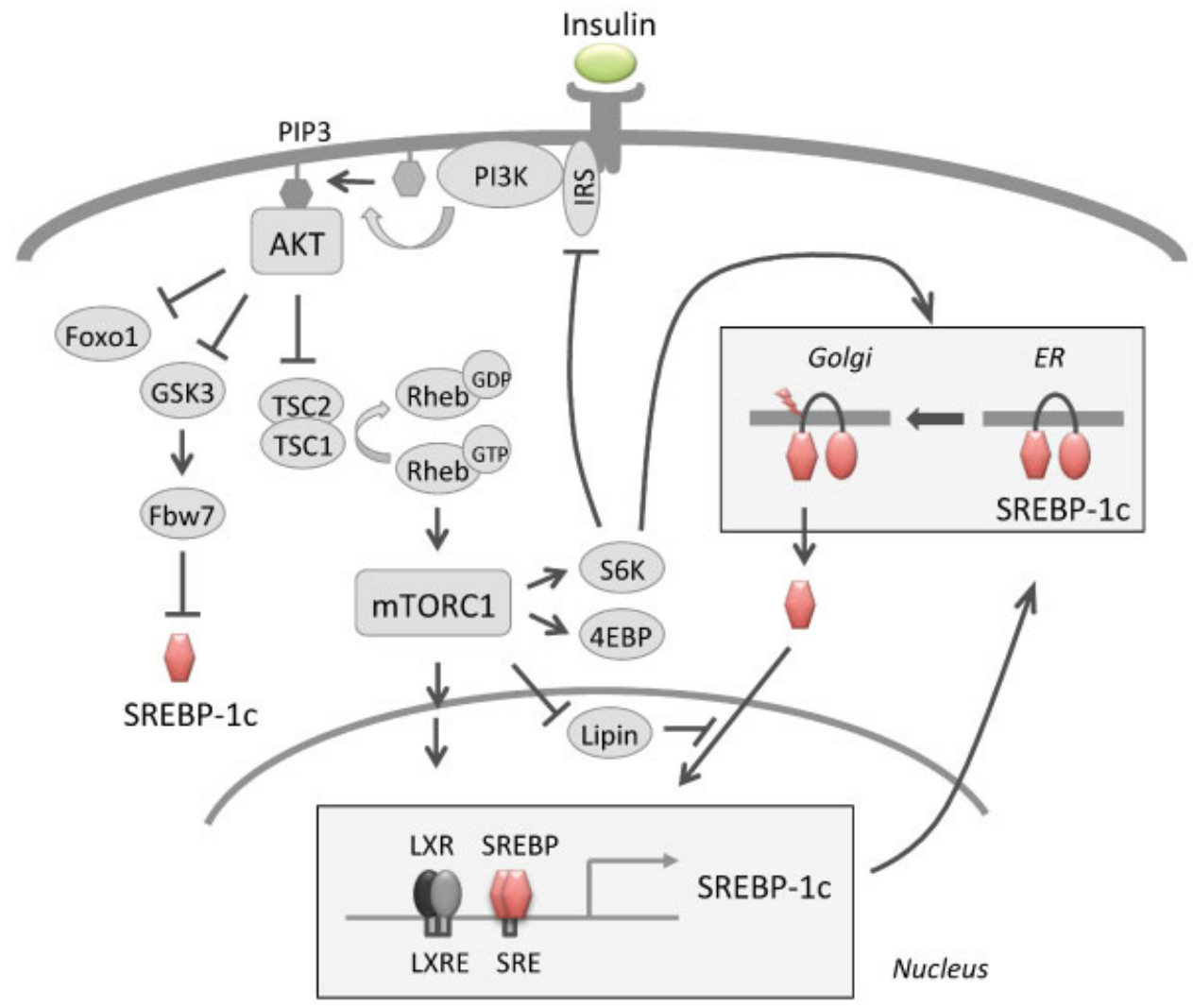

Fig. 3 Regulation of sterol regulatory element binding protein (SREBP) by the insulin signaling pathway. Insulin activates SREBP-1 through multiple mechanisms. Insulin stimulates SREBP-1c transcription, promotes proteolytic processing, facilitates the nuclear import of the processed protein, and suppresses the proteasomal degradation of SREBP-1. 
fasting followed by refeeding with a high-carbohydrate diet, uncovering a critical role of the mTORC1 pathway in the insulin-induced lipogenesis program. ${ }^{43}$ Similarly, insulin-induced SREBP-1c mRNA expression was abolished by rapamycin or small molecule inhibitors of PI3K or AKT that block mTORC1 activation in cultured rat hepatocytes. ${ }^{43}$ mTORC1 protein kinase directly phosphorylates two major downstream targets, initiation factor $4 \mathrm{E}$-binding protein (4E-BP) and p70 ribosomal S6 kinase (p70S6K), increasing mRNA translation. ${ }^{41,45,46}$ p70S6K has a growing number of downstream targets in addition to the ribosomal protein S6. ${ }^{47,48}$ Notably, a p70S6K inhibitor had no effect on insulin-induce SREBP-1c mRNA expression, ${ }^{43}$ but inhibited the proteolytic processing of SREBP-1c protein, ${ }^{26}$ indicating that mTORC1 regulates SREBP-c mRNA expression and protein processing through distinct mechanisms.

Although the acute inhibition of mTORC1 activity suppresses insulin-induced SREBP-1c mRNA expression and lipogenic gene expression, constitutive activation of mTORC1 ironically suppresses SREBP-1c activation. ${ }^{27}$ Genetic ablation of TSC1 in the liver caused constitutive activation of MTORC1, but suppressed age- and diet-induced hepatic steatosis, possibly due to defective SREBP-1c expression. This unexpected phenotype reflects the complex feedback regulation of the insulin signaling pathway leading to SREBP-1c activation. ${ }^{12,40}$ It has been well established that MTORC1 negatively regulates insulin signaling at multiple steps of the signaling cascade as feedback mechanisms. ${ }^{48-50}$ Indeed, AKT was markedly suppressed in TSC1 deficient hepatocytes, which might contribute to the decreased expression of SREBP-1c and lipogenic genes. An important question that remains to be answered is what ultimately increases SREBP-1c transcription in response to insulin stimulation. So far, liver $\mathrm{X}$ receptors (LXRs) and SREBP-1c itself are known to activate SREBP-1c promoter. $^{51-55}$ The relative contribution of LXR and SREBP-1c in the insulin-induced transcriptional activation of SREBP-1c remains to be further investigated.

Liver $\mathrm{X}$ receptors are members of the nuclear hormone receptor superfamily that play a critical role in cholesterol efflux, excretion, and absorption. ${ }^{56}$ LXR $\alpha$ also plays an important role in fatty acid and triglyceride synthesis, as it induces SREBP-1c expression via an LXR response element on its promoter. ${ }^{51,53,57}$ The lipogenic activity of LXR $\alpha$ was abrogated in SREBP-1c deficient mice, indicating that LXR promotes lipogenesis through SREBP-1c. ${ }^{4}$ LXR $\alpha$ - deficient mice exhibit reduced expression of SREBP-1C and lipogenic genes such as SCD1 and FAS in the liver. ${ }^{53,54,58,59}$ In contrast, a highcholesterol diet or the LXR $\alpha$ agonist TO-901317 increase SREBP-1c and stimulate lipogenesis in the liver. ${ }^{53,54,58,59}$ Importantly, disruption of LXR-binding sites on the SREBP1c promoter abolished the induction of promoter activity by insulin or TO-901317, suggesting that LXR $\alpha$ is responsible for SREBP-1c induction in response to insulin. ${ }^{51}$ However, it is not known how insulin activates LXR $\alpha$. The role of LXR $\alpha$ in the insulin signaling cascade appears to be specific to SREBP-1c because insulin does not induce other LXR $\alpha$ target genes. ${ }^{60,61}$ It has been reported that insulin modestly increases LXR $\alpha$ mRNA in cultured rat hepatocytes. ${ }^{62}$ It is also possible that insulin stimulates the production of LXR $\alpha$ ligands to activate LXR $\alpha$.

\section{Regulation of SREBP Protein Stability}

The nuclear form of SREBP protein is highly unstable, as it is degraded via ubiquitin-dependent proteasomal degradation pathways. $^{63,64}$ Treatment of proteasome inhibitors increases the amount of nuclear SREBP, but not the precursor form, indicating that only the processed nuclear forms of SREBPs are subjected to proteasomal degradation. Ubiquitination and degradation of SREBPs are closely associated with their transcriptional activities. ${ }^{65}$ Inhibition of transcriptional activity of SREBPS by mutating critical functional domains, or by treating with RNA polymerase inhibitor prevented the degradation of SREBPs. It is conceivable that the proteasomal degradation of SREBPs portrays a feedback regulation of SREBP activity to fine tune the transcriptional response of lipogenesis. Ubiquitination of SREBP could be suppressed by SREBP coactivators, CREB-binding protein (CBP) and p300, which competitively acetylate the lysine residue that is also targeted by ubiquitination, leading to stabilization of SREBP and the induction of SREBP target genes (LDLR, HMG-CoA reductase) and sterol synthesis. ${ }^{66}$

Phosphorylation of SREBP is critically required for its ubiquitination. F-box and WD repeat domain-containing 7 (Fbw7) is a cullin-RING type E3 ubiquitin ligase that has emerged as the major ubiquitin ligase for SREBPs. Phosphorylation of SREBP induces its interaction with Fbw7, and thus facilitates its ubiquitination and degradation. ${ }^{67,68}$ GSK3 phosphorylates SREBP-1a at T426 and S430 residues, which resemble Cdc4 phosphodegron (CPD) motif, a recognition site for Fbw7. SREBP-1c and SREBP-2 are also similarly ubiquitinated by Fbw7. DNA binding of SREBP facilitates the recruitment of GSK3 to the promoter, and the subsequent interaction between SREBP1 and GSK3. ${ }^{69}$ Insulin regulates the stability of SREBP by controlling its phosphorylation by GSK3 and interaction with Fbw7. ${ }^{70}$ Insulin-mediated AKT activation induces Ser-9 phosphorylation of GSK3, leading to the suppression of its kinase activity. ${ }^{71}$ Consequently, insulin suppresses SREBP phosphorylation and the following Fbw7-dependent degradation. Cyclin-dependent kinase 8 (CDK8) can also phosphorylate SREBP-1c, and thus trigger its ubiquitination by Fbw7 and proteasomal degradation. ${ }^{72}$ As CDK8 expression is suppressed by insulin, CDK8-triggered SREBP-1c ubiquitination/degradation would constitute a regulatory mechanism of the lipogenesis program. Indeed, knockdown of CDK8 in mouse liver increased SREBP-1c target genes (FAS, ACS, and SCD1) and hepatic triglyceride levels. ${ }^{72}$

Fbw7 deficiency stabilizes nuclear SREBPs and enhances the expression of their target genes, leading to increased synthesis of fatty acids, TG, and cholesterol, and increased receptor-mediated uptake of low-density lipoprotein (LDL). ${ }^{73}$ Liver-specific deletion of Fbw7 in vivo increased the expression of hepatic SREBP-1c and lipogenic genes, which was accompanied by massive lipid deposition and the occurrence of nonalcoholic steatohepatitis (NASH) in the mutant mice. ${ }^{73}$ These findings establish Fbw7 as an important regulator of SREBP protein stability and lipid metabolism. 


\section{MicroRNA-SREBP Connection in Lipid Metabolism}

MicroRNAs (miRNAs) are small nonprotein-coding RNAs of $\sim$ $23 \mathrm{nt}$ in length that are produced from longer primary miRNA transcripts via sequential processing by DROSHA and DICER ribonucleases. ${ }^{74}$ miRNAs bind to the 3 ' untranslated regions of target mRNAs, and thereby either promote the degradation or suppress the translation of target mRNAs. ${ }^{74}$ Given that a single miRNA can control the expression of multiple target genes in the same pathway, miRNAs have emerged as critical regulators of a variety of biological processes, including nutrient metabolism. . $^{7,76}$

Interestingly, recent reports revealed that SREBP genes (SREBF1 and SREBF2) harbor miRNAs within introns that are consequently cotranscribed with the respective SREBP genes. In humans, miR-33a is located in intron 16 of the SREBF2 gene (encoding SREBP-2), and miR-33b is within intron 17 of the SREBF1 gene (encoding SREBP-1a and SREBP-1c). ${ }^{77-81}$ Mature miR-33a and miR-33b have similar nucleotide sequences and hence would be expected to regulate overlapping target mRNAs. Although miR-33a is evolutionarily conserved in multiple animal species, miR-33b exists in the human, but is absent in the rodent genome. ${ }^{79,81}$ Reminiscent of the critical role of SREBP proteins in lipid metabolism, miR-33a and miR-33b also regulate cholesterol and fatty acid homeostasis. ${ }^{77-81}$ miRNA target sequence analysis predicted that miR-33a and miR-33b target adenosine triphosphate-binding cassette A1 (ABCA1) mRNA, which encodes a cholesterol transporter that plays a crucial role in cholesterol efflux. Indeed, silencing or genetic ablation of miR-33 markedly increased ABCA1 expression both in cultured hepatocytes and macrophages, and increased plasma high-density lipoprotein (HDL) levels. ${ }^{77-82}$

Given the beneficial effects of miR-33 antagonism in increasing plasma HDL levels, miR-33 inhibition aroused great interest as a potential therapeutic approach to treat cardiovascular diseases. ${ }^{83}$ Indeed, Rayner et al demonstrated that anti-miR-33 treatment promoted reverse cholesterol transport and reduced atherosclerotic plaques in LDL-receptor knockout $\left(\mathrm{Ldll}^{-l-}\right)$ mice. ${ }^{84}$ Similarly, genetic loss of miR33 in ApoE null mice increased circulating HDL-cholesterol levels and reduced plaque size. ${ }^{85} \mathrm{~A}$ recent study in nonhuman primates also reported the increase of HDL cholesterol by anti-miR-33 therapy, highlighting the strong potential of anti-miR-33 as a new therapy for coronary heart disease. ${ }^{86}$ However, subsequent independent studies using anti-miR-33 antisense oligonucleotides or locked nucleic acids reported somewhat inconsistent effects of miR-33 inhibition on HDL cholesterol levels and atherosclerotic lesion development in $\mathrm{Ldll}^{-1-}$ mice. ${ }^{87,88}$ For example, both studies found that antimiR-33 had no effect on HDL cholesterol levels in $\mathrm{Ldll}^{-1-}$ mice fed a Western diet, although animals on a chow diet exhibited increased HDL cholesterol by anti-miR-33 treatment. Nonetheless, Rotllan et al demonstrated that anti-miR-33 therapy significantly reduced atherosclerotic lesions and macrophage infiltration. ${ }^{88}$ In contrast, Marquart et al failed to detect any significant changes in the size or composition of atheroscle- rotic plaques in anti-miR-33 treated mice, while plasma TG levels were significantly increased. ${ }^{87}$ Further studies should address the effectiveness of anti-miR-33 treatment as a therapeutic approach, and identify the full spectrum of miR-33 target mRNAs.

A recent elegant study identified a pair of microRNAs that are transcriptionally induced by SREBPs, and in turn suppress SREBPs, constituting a negative feedback loop. ${ }^{89}$ Sterol regulatory element binding proteins directly activate the transcription of a primary miRNA transcript that is processed to three miRNAs; miR-96, miR-182, and miR-183. Interestingly, miR-96 and miR-182 suppressed the expression of the processed SREBPs and the synthesis of fatty acids and cholesterol, suggesting that these miRNAs regulate the processing or stability of SREBPs. Target sequence analysis predicted that Insig-2 and Fbw7, which regulate the processing and proteasomal degradation of SREBPs, might be regulated by miR-96 and miR-182, respectively. Indeed, miR-96 and miR-182 suppressed the synthesis of Insig- 2 and Fbw7, and increased the processed SREBP1 and SREBP2 protein levels. This study reveals a new layer of regulatory mechanisms in lipid metabolism.

\section{Carbohydrate Response Element Binding Protein}

Carbohydrate response element binding protein (ChREBP) was first identified as a glucose responsive transcription factor, which regulates glycolytic, gluconeogenic, and lipogenic gene expression. ${ }^{6,7}$ Transcriptional targets of ChREBP encode important enzymes in these pathways including Lpyruvate kinase (L-PK) for glycolysis, glucose 6 phosphatase catalytic subunit (G6PC) for gluconeogenesis, fatty acid synthase (FAS), acetyl coA carboxylase 1 (ACC1), and stearyl coA desaturase 1 (SCD 1) for lipogenesis. ${ }^{6}$ Carbohydrate-response elements (ChoREs) have been identified in promoters of these genes, which are composed of two E-box (CACGTG) or E-boxlike sequences separated by five nucleotides. ${ }^{90,91}$ Carbohydrate response element binding protein and its interaction partner Max-like protein X (MLX) form heterodimers and bind to the ChoREs to induce the expression of its target genes (-Fig. 4). ${ }^{92,93}$

Carbohydrate response element binding protein contains two nuclear export signals and one nuclear localization signal near the N-terminal, proline-rich domains, a basic loop-helixleucine-zipper (b/HLH/Zip), and a leucine-zipper-like (Ziplike) domain., ${ }^{7}, 94$

Posttranslational modification of ChREBP is required for its activation. The phosphorylation/dephosphorylation of ChREBP has been proposed to be important for ChREBP nuclear translocation and activation. Under basal conditions, like starvation or low-glucose concentrations, ChREBP is phosphorylated on Ser-196, Ser-626, and Thr-66 by cAMPdependent protein kinase (PKA), on Ser568 by AMP-activated protein kinase (AMPK) and localized in the cytosol. ${ }^{8,95}$ Upon high-glucose stimulation, xylulose 5-phosphate (X5P), an intermediate of the pentose phosphate pathway, activates protein phosphatase 2A (PP2A) and dephosphorylates 


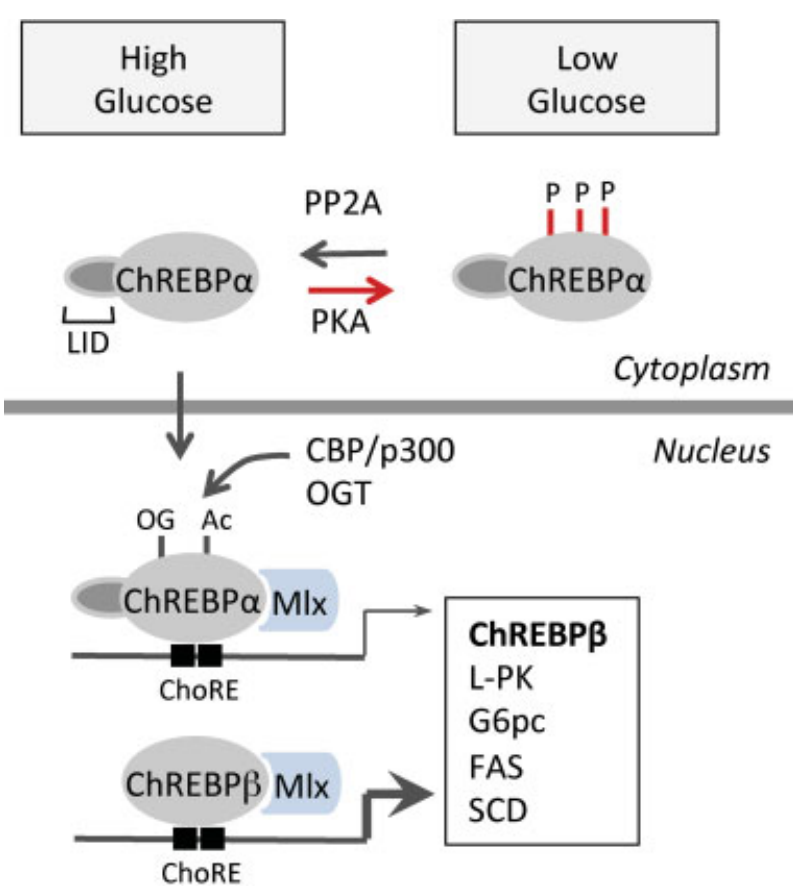

Fig. 4 Regulation of carbohydrate response element binding protein (ChREBP) activity. The phosphorylation/dephosphorylation of ChREBP $\alpha$ by PKA/protein phosphatase 2A (PP2A) is involved in ChREBP $\alpha$ nuclear translocation and activation. Acetylation by coactivator CBP/P300 and O-GlcNAcylation by O-GlcNAc transferase (OGT) also contribute to ChREBP $\alpha$ transcriptional activities. ChREBP $\alpha$ forms a heterodimer with Max-like protein X (MLX) and binds to the carbohydrate-response elements (ChoREs) in the nucleus to induce its target genes involved in glycolytic and lipogenic pathways. In adipose tissue, active ChREBPa induces expression of ChREBPB, a new ChREBP isoform that lacks the low-glucose inhibitory domain (LID), and is hence constitutively active regardless of glucose concentration.

ChREBP, allowing its translocation into the nucleus and activation. ${ }^{96}$ However, some studies show that mutation of one or several PKA phosphorylation sites did not affect the responsiveness of ChREBP to high-glucose levels, suggesting a more complex mechanism could be involved. ${ }^{97}$ Transactivity of ChREBP can also be modulated through acetylation on Lys672 by histone acetyl-transferase (HAT) and its coactivator p300 ${ }^{98}$ and by $O$-linked- $\beta-N$-actetylation. ${ }^{99,100}$

Alternatively, intramolecular interaction has been proposed to be another way to modulate ChREBP activity. Carbohydrate response element binding protein contains a glucose-sensing module near the $\mathrm{N}$-terminus, which consists of a low-glucose inhibitory domain (LID) and a glucose response activation conserved element (GRACE). Due to the inhibition of the LID domain on GRACE, ChREBP is restrained in an unfavorable conformation for DNA binding and activation, which is reversed by high glucose..$^{94,101-103}$ In line with this model, deletion of the LID domain produced a constitutively active ChREBP even under low-glucose conditions. ${ }^{102}$ The involvement of the glucose-sensing module and conformational modulation has been implicated in the regulation of ChREBP activity by glucose metabolites, such as glucose 6 phosphate (G6P). ${ }^{104,105}$

The mechanism of carbohydrate-mediated ChREBP activation may involve feed-forward regulation because changes of ChREBP activity can also be reflected on ChREBP mRNA levels. ${ }^{106,107}$ Recently, the self-regulation of ChREBP in adipose tissue has been revealed with the discovery of a novel ChREBP isoform, ChREBP $\beta^{108}$ ChREBP $\beta$ is transcribed from an alternative promoter, differing from the previously identified ChREBP, or ChREBP $\alpha$. ChREBP $\beta$ protein does not contain LID and nucleus export signals, therefore exhibits constitutively higher transactivation ability than ChREBP $\alpha$ with increased nuclear localization, ${ }^{102}$ regardless of the glucose concentration. ${ }^{108}$ ChREBP $\beta$ expression was markedly increased by cotransfection of ChREBP $\alpha$ and LMX in a glucose dose-dependent manner. The ChoREs are also identified in the promoter region of ChREBP $\beta$ and the deletion of these elements completely abolished the responsiveness of the ChREBP $\beta$ promoter to ChREBP $\alpha /$ MLX. ${ }^{108}$ Therefore, ChREBP $\alpha$ may be activated by high-glucose concentrations as previously reported, and induce ChREBP $\beta$ expression as a feedforward regulation. It remains to be determined if this feedforward regulation of ChREBP also occurs in the liver and other tissues.

As ChREBP directly regulates genes involved in both glucose and lipid metabolism that indirectly influence each other, genetic manipulation of ChREBP expression in vivo results in rather complex metabolic changes. ChREBP ${ }^{-1-}$ mice have impaired glycolytic and lipogenic pathways in the liver and show moderate glucose intolerance. ${ }^{6}$ Global or liver-specific deletion of ChREBP greatly ameliorated fatty liver diseases, and improved overall glucose tolerance and insulin sensitivity in ob/ob mice, possibly through decreasing de novo lipogenesis. ${ }^{109,110}$ Overexpression of ChREBP in the liver increased hepatic steatosis associated with the increased expression of genes regulating fatty acid and TG synthesis in the liver. Interestingly, ChREBP transgenic mice exhibited elevated monounsaturated fatty acids in the liver, which conferred improved glucose tolerance and insulin sensitivity upon high-fat-diet feeding despite greater hepatic steatosis. ${ }^{111}$

Carbohydrate response element binding protein expression is induced during adipocyte differentiation, and by refeeding with a high-carbohydrate diet in adipose tissue, suggesting it might have a metabolic function in adipocytes. ${ }^{112}$ Indeed, a recent study demonstrated that ChREBP regulates the de novo lipogenesis program in response to glucose flux in adipocytes, and that adipose tissue ChREBP levels correlate well with glucose tolerance and insulin sensitivity in humans. ${ }^{108}$ Carbohydrate response element binding protein is also expressed in pancreatic $\beta$ cells. Glucose stimulated the expression of ChREBP target genes in $\beta$ cells, ${ }^{107,113}$ and activation of ChREBP promoted glucose stimulated $\beta$-cell proliferation. ${ }^{114}$

\section{Concluding Remarks}

In this review, we summarized the complex signaling network that controls the hepatic lipogenesis transcriptional program activated directly or indirectly by carbohydrate ingestion. Sterol regulatory element binding protein and ChREBP are major transcriptional regulators that are activated by carbohydrate signal, and stimulate de novo hepatic 
lipogenesis. Recent studies revealed that AKT/mTORC1 signaling pathways are critically involved not only in the transcriptional activation, but also in the posttranslational processing of SREBP-1c. Uncontrolled de novo lipogenesis causes hepatic steatosis, which is closely associated with the onset of obesity, insulin resistance, and type 2 diabetes. Excessive lipogenesis induced by transgenic overexpression of SREBP-1, ingestion of a high-fructose diet, or leptin deficiency causes hepatic steatosis. Under insulin-resistant states, hyperinsulinemia can also activate SREBP-1 to induce hepatic steatosis, with the loss of insulin-mediated suppression of gluconeogenesis. ${ }^{15}$ On the other hand, inhibition of SREBP-1 could be a potential therapeutic approach to treat dyslipidemia and the metabolic syndrome. A better understanding of the signaling pathways controlling lipogenesis may lead to the identification of novel targets for metabolic diseases.

The role of ChREBP in sensing glucose and regulating nutrient homeostasis, especially lipid synthesis, is of great interest, considering its therapeutic potential in the treatment of diabetes and metabolic syndrome. With the discovery of ChREBP $\alpha$ and $\beta$ isoforms, tissue-specific distributions of ChREBP should be more carefully considered when studying its regulation on lipid metabolism and systemic glucose homeostasis.

\section{Acknowledgments}

This work was supported by NIH grant DK089211 to AnnHwee Lee.

\section{References}

1 Towle HC. Glucose as a regulator of eukaryotic gene transcription. Trends Endocrinol Metab 2005;16(10):489-494

2 Horton JD, Bashmakov Y, Shimomura I, Shimano H. Regulation of sterol regulatory element binding proteins in livers of fasted and refed mice. Proc Natl Acad Sci U S A 1998;95(11):5987-5992

3 Kim JB, Sarraf P, Wright M, et al. Nutritional and insulin regulation of fatty acid synthetase and leptin gene expression through ADD1/SREBP1. J Clin Invest 1998;101(1):1-9

4 Liang G, Yang J, Horton JD, Hammer RE, Goldstein JL, Brown MS. Diminished hepatic response to fasting/refeeding and liver $\mathrm{X}$ receptor agonists in mice with selective deficiency of sterol regulatory element-binding protein-1c. J Biol Chem 2002; 277(11):9520-9528

5 Horton JD, Shah NA, Warrington JA, et al. Combined analysis of oligonucleotide microarray data from transgenic and knockout mice identifies direct SREBP target genes. Proc Natl Acad Sci U S A 2003;100(21):12027-12032

6 Iizuka K, Bruick RK, Liang G, Horton JD, Uyeda K. Deficiency of carbohydrate response element-binding protein (ChREBP) reduces lipogenesis as well as glycolysis. Proc Natl Acad Sci U S A 2004;101(19):7281-7286

7 Yamashita H, Takenoshita M, Sakurai M, et al. A glucose-responsive transcription factor that regulates carbohydrate metabolism in the liver. Proc Natl Acad Sci U S A 2001;98(16):9116-9121

8 Kawaguchi T, Takenoshita M, Kabashima T, Uyeda K. Glucose and cAMP regulate the L-type pyruvate kinase gene by phosphorylation/dephosphorylation of the carbohydrate response element binding protein. Proc Natl Acad Sci U S A 2001;98(24): 13710-13715
9 Ma L, Robinson LN, Towle HC. ChREBP*Mlx is the principal mediator of glucose-induced gene expression in the liver. J Biol Chem 2006;281(39):28721-28730

10 Horton JD, Goldstein JL, Brown MS. SREBPs: activators of the complete program of cholesterol and fatty acid synthesis in the liver. J Clin Invest 2002;109(9):1125-1131

11 Ferré P, Foufelle F. Hepatic steatosis: a role for de novo lipogenesis and the transcription factor SREBP-1c. Diabetes Obes Metab 2010;12(Suppl 2):83-92

12 Jeon TI, Osborne TF. SREBPs: metabolic integrators in physiology and metabolism. Trends Endocrinol Metab 2012;23(2):65-72

13 Hua X, Wu J, Goldstein JL, Brown MS, Hobbs HH. Structure of the human gene encoding sterol regulatory element binding protein1 (SREBF1) and localization of SREBF1 and SREBF2 to chromosomes 17p11.2 and 22q13. Genomics 1995;25(3):667-673

14 Shimomura I, Shimano H, Horton JD, Goldstein JL, Brown MS Differential expression of exons 1a and 1c in mRNAs for sterol regulatory element binding protein-1 in human and mouse organs and cultured cells. J Clin Invest 1997;99(5):838-845

15 Im SS, Yousef L, Blaschitz C, et al. Linking lipid metabolism to the innate immune response in macrophages through sterol regulatory element binding protein-1a. Cell Metab 2011;13(5):540-549

16 Brown MS, Goldstein JL. The SREBP pathway: regulation of cholesterol metabolism by proteolysis of a membrane-bound transcription factor. Cell 1997;89(3):331-340

17 Goldstein JL, Rawson RB, Brown MS. Mutant mammalian cells as tools to delineate the sterol regulatory element-binding protein pathway for feedback regulation of lipid synthesis. Arch Biochem Biophys 2002;397(2):139-148

18 Sun LP, Li L, Goldstein JL, Brown MS. Insig required for sterolmediated inhibition of Scap/SREBP binding to COPII proteins in vitro. J Biol Chem 2005;280(28):26483-26490

19 Adams CM, Goldstein JL, Brown MS. Cholesterol-induced conformational change in SCAP enhanced by Insig proteins and mimicked by cationic amphiphiles. Proc Natl Acad Sci U S A 2003; 100(19):10647-10652

20 Hua X, Nohturfft A, Goldstein JL, Brown MS. Sterol resistance in $\mathrm{CHO}$ cells traced to point mutation in SREBP cleavage-activating protein. Cell 1996;87(3):415-426

21 Sun LP, Seemann J, Goldstein JL, Brown MS. Sterol-regulated transport of SREBPs from endoplasmic reticulum to Golgi: Insig renders sorting signal in Scap inaccessible to COPII proteins. Proc Natl Acad Sci U S A 2007;104(16):6519-6526

22 Radhakrishnan A, Goldstein JL, McDonald JG, Brown MS. Switchlike control of SREBP-2 transport triggered by small changes in ER cholesterol: a delicate balance. Cell Metab 2008;8(6):512-521

23 Shimomura I, Bashmakov Y, Ikemoto S, Horton JD, Brown MS, Goldstein JL. Insulin selectively increases SREBP-1c mRNA in the livers of rats with streptozotocin-induced diabetes. Proc Natl Acad Sci U S A 1999;96(24):13656-13661

24 Hegarty BD, Bobard A, Hainault I, Ferré P, Bossard P, Foufelle F. Distinct roles of insulin and liver $\mathrm{X}$ receptor in the induction and cleavage of sterol regulatory element-binding protein-1c. Proc Natl Acad Sci U S A 2005;102(3):791-796

25 Yellaturu CR, Deng X, Cagen LM, et al. Insulin enhances posttranslational processing of nascent SREBP-1c by promoting its phosphorylation and association with COPII vesicles. J Biol Chem 2009;284(12):7518-7532

26 Owen JL, Zhang Y, Bae SH, et al. Insulin stimulation of SREBP-1c processing in transgenic rat hepatocytes requires p70 S6-kinase. Proc Natl Acad Sci U S A 2012;109(40):16184-16189

27 Yecies JL, Zhang HH, Menon S, et al. Akt stimulates hepatic SREBP1c and lipogenesis through parallel mTORC1-dependent and independent pathways. Cell Metab 2011;14(1):21-32

28 Yabe D, Komuro R, Liang G, Goldstein JL, Brown MS. Liver-specific mRNA for Insig-2 down-regulated by insulin: implications for fatty acid synthesis. Proc Natl Acad Sci U S A 2003;100(6): 3155-3160 
29 Yellaturu CR, Deng X, Park EA, Raghow R, Elam MB. Insulin enhances the biogenesis of nuclear sterol regulatory elementbinding protein (SREBP)-1c by posttranscriptional down-regulation of Insig-2A and its dissociation from SREBP cleavage-activating protein (SCAP).SREBP-1c complex. J Biol Chem 2009;284(46): 31726-31734

30 Nagoshi E, Imamoto N, Sato R, Yoneda Y. Nuclear import of sterol regulatory element-binding protein-2, a basic helix-loop-helixleucine zipper (bHLH-Zip)-containing transcription factor, occurs through the direct interaction of importin beta with HLH-Zip. Mol Biol Cell 1999;10(7):2221-2233

31 Peterson TR, Sengupta SS, Harris TE, et al. mTOR complex 1 regulates lipin 1 localization to control the SREBP pathway. Cell 2011;146(3):408-420

32 Donkor J, Sariahmetoglu M, Dewald J, Brindley DN, Reue K. Three mammalian lipins act as phosphatidate phosphatases with distinct tissue expression patterns. J Biol Chem 2007;282(6): 3450-3457

33 Harris TE, Huffman TA, Chi A, et al. Insulin controls subcellular localization and multisite phosphorylation of the phosphatidic acid phosphatase, lipin 1. J Biol Chem 2007;282(1):277-286

34 Finck BN, Gropler MC, Chen Z, et al. Lipin 1 is an inducible amplifier of the hepatic PGC-1alpha/PPARalpha regulatory pathway. Cell Metab 2006;4(3):199-210

35 Foretz M, Pacot C, Dugail I, et al. ADD1/SREBP-1c is required in the activation of hepatic lipogenic gene expression by glucose. Mol Cell Biol 1999;19(5):3760-3768

36 Tang X, Ma H, Shen Z, Zou S, Xu X, Lin C. Dehydroepiandrosterone activates cyclic adenosine $3^{\prime}, 5^{\prime}$-monophosphate/protein kinase $\mathrm{A}$ signalling and suppresses sterol regulatory element-binding protein-1 expression in cultured primary chicken hepatocytes. Br J Nutr 2009;102(5):680-686

37 Manning BD, Cantley LC. AKT/PKB signaling: navigating downstream. Cell 2007;129(7):1261-1274

38 Lizcano JM, Alessi DR. The insulin signalling pathway. Curr Biol 2002;12(7):R236-R238

39 Biddinger SB, Kahn CR. From mice to men: insights into the insulin resistance syndromes. Annu Rev Physiol 2006; 68:123-158

40 Howell JJ, Manning BD. mTOR couples cellular nutrient sensing to organismal metabolic homeostasis. Trends Endocrinol Metab 2011;22(3):94-102

41 Guertin DA, Sabatini DM. Defining the role of mTOR in cancer. Cancer Cell 2007;12(1):9-22

42 Laplante M, Sabatini DM. An emerging role of mTOR in lipid biosynthesis. Curr Biol 2009;19(22):R1046-R1052

43 Li S, Brown MS, Goldstein JL. Bifurcation of insulin signaling pathway in rat liver: mTORC1 required for stimulation of lipogenesis, but not inhibition of gluconeogenesis. Proc Natl Acad Sci U S A 2010;107(8):3441-3446

44 Porstmann T, Santos CR, Griffiths B, et al. SREBP activity is regulated by mTORC1 and contributes to Akt-dependent cell growth. Cell Metab 2008;8(3):224-236

45 Brown EJ, Beal PA, Keith CT, Chen J, Shin TB, Schreiber SL. Control of p70 s6 kinase by kinase activity of FRAP in vivo. Nature 1995; 377(6548):441-446

46 Brunn GJ, Hudson CC, Sekulić A, et al. Phosphorylation of the translational repressor PHAS-I by the mammalian target of rapamycin. Science 1997;277(5322):99-101

47 Zoncu R, Efeyan A, Sabatini DM. mTOR: from growth signal integration to cancer, diabetes and ageing. Nat Rev Mol Cell Biol 2011;12(1):21-35

$48 \mathrm{Um} \mathrm{SH}$, Frigerio F, Watanabe M, et al. Absence of S6K1 protects against age- and diet-induced obesity while enhancing insulin sensitivity. Nature 2004;431(7005):200-205

49 Yu Y, Yoon SO, Poulogiannis G, et al. Phosphoproteomic analysis identifies Grb10 as an mTORC1 substrate that negatively regulates insulin signaling. Science 2011;332(6035):1322-1326
50 Hsu PP, Kang SA, Rameseder J, et al. The mTOR-regulated phosphoproteome reveals a mechanism of mTORC1-mediated inhibition of growth factor signaling. Science 2011;332(6035): $1317-1322$

51 Chen G, Liang G, Ou J, Goldstein JL, Brown MS. Central role for liver $\mathrm{X}$ receptor in insulin-mediated activation of Srebp-1c transcription and stimulation of fatty acid synthesis in liver. Proc Natl Acad Sci U S A 2004;101(31):11245-11250

52 Chen W, Chen G, Head DL, Mangelsdorf DJ, Russell DW. Enzymatic reduction of oxysterols impairs LXR signaling in cultured cells and the livers of mice. Cell Metab 2007;5(1):73-79

53 Repa JJ, Liang G, Ou J, et al. Regulation of mouse sterol regulatory element-binding protein-1c gene (SREBP-1c) by oxysterol receptors, LXRalpha and LXRbeta. Genes Dev 2000;14(22):2819-2830

54 Zhang Y, Breevoort SR, Angdisen J, et al. Liver LXR $\alpha$ expression is crucial for whole body cholesterol homeostasis and reverse cholesterol transport in mice. J Clin Invest 2012;122(5): 1688-1699

55 Amemiya-Kudo M, Shimano H, Yoshikawa T, et al. Promoter analysis of the mouse sterol regulatory element-binding protein-1c gene. J Biol Chem 2000;275(40):31078-31085

56 Calkin AC, Tontonoz P. Liver X receptor signaling pathways and atherosclerosis. Arterioscler Thromb Vasc Biol 2010;30(8): 1513-1518

57 Yoshikawa T, Shimano H, Amemiya-Kudo M, et al. Identification of liver $\mathrm{X}$ receptor-retinoid $\mathrm{X}$ receptor as an activator of the sterol regulatory element-binding protein 1c gene promoter. Mol Cell Biol 2001;21(9):2991-3000

58 Peet DJ, Turley SD, Ma W, et al. Cholesterol and bile acid metabolism are impaired in mice lacking the nuclear oxysterol receptor LXR alpha. Cell 1998;93(5):693-704

59 Kalaany NY, Gauthier KC, Zavacki AM, et al. LXRs regulate the balance between fat storage and oxidation. Cell Metab 2005;1(4): 231-244

60 Kok T, Wolters H, Bloks VW, et al. Induction of hepatic ABC transporter expression is part of the PPARalpha-mediated fasting response in the mouse. Gastroenterology 2003;124(1):160-171

61 Bloks VW, Bakker-Van Waarde WM, Verkade HJ, et al. Downregulation of hepatic and intestinal Abcg5 and Abcg8 expression associated with altered sterol fluxes in rats with streptozotocininduced diabetes. Diabetologia 2004;47(1):104-112

62 Tobin KA, Ulven SM, Schuster GU, et al. Liver X receptors as insulin-mediating factors in fatty acid and cholesterol biosynthesis. J Biol Chem 2002;277(12):10691-10697

63 Wang X, Sato R, Brown MS, Hua X, Goldstein JL. SREBP-1, a membrane-bound transcription factor released by sterol-regulated proteolysis. Cell 1994;77(1):53-62

64 Hirano Y, Yoshida M, Shimizu M, Sato R. Direct demonstration of rapid degradation of nuclear sterol regulatory element-binding proteins by the ubiquitin-proteasome pathway. J Biol Chem 2001; 276(39):36431-36437

65 Sundqvist A, Ericsson J. Transcription-dependent degradation controls the stability of the SREBP family of transcription factors. Proc Natl Acad Sci U S A 2003;100(24):13833-13838

66 Ericsson J, Edwards PA. CBP is required for sterol-regulated and sterol regulatory element-binding protein-regulated transcription. J Biol Chem 1998;273(28):17865-17870

67 Kim KH, Song MJ, Yoo EJ, Choe SS, Park SD, Kim JB. Regulatory role of glycogen synthase kinase 3 for transcriptional activity of ADD1/SREBP1c. J Biol Chem 2004;279(50):51999-52006

68 Sundqvist A, Bengoechea-Alonso MT, Ye X, et al. Control of lipid metabolism by phosphorylation-dependent degradation of the SREBP family of transcription factors by SCF(Fbw7). Cell Metab 2005;1(6):379-391

69 Punga T, Bengoechea-Alonso MT, Ericsson J. Phosphorylation and ubiquitination of the transcription factor sterol regulatory element-binding protein-1 in response to DNA binding. J Biol Chem 2006;281(35):25278-25286 
70 Krycer JR, Sharpe LJ, Luu W, Brown AJ. The Akt-SREBP nexus: cell signaling meets lipid metabolism. Trends Endocrinol Metab 2010;21(5):268-276

71 Cross DAE, Alessi DR, Cohen P, Andjelkovich M, Hemmings BA. Inhibition of glycogen synthase kinase- 3 by insulin mediated by protein kinase B. Nature 1995;378(6559):785-789

72 Zhao X, Feng D, Wang $Q$ et al. Regulation of lipogenesis by cyclindependent kinase 8-mediated control of SREBP-1. J Clin Invest 2012;122(7):2417-2427

73 Onoyama I, Suzuki A, Matsumoto A, et al. Fbxw7 regulates lipid metabolism and cell fate decisions in the mouse liver. J Clin Invest 2011;121(1):342-354

74 Bartel DP. MicroRNAs: target recognition and regulatory functions. Cell 2009;136(2):215-233

75 Fernández-Hernando C, Ramírez CM, Goedeke L, Suárez Y. MicroRNAs in metabolic disease. Arterioscler Thromb Vasc Biol 2013; 33(2):178-185

76 Rottiers V, Näär AM. MicroRNAs in metabolism and metabolic disorders. Nat Rev Mol Cell Biol 2012;13(4):239-250

77 Dávalos A, Goedeke L, Smibert P, et al. miR-33a/b contribute to the regulation of fatty acid metabolism and insulin signaling. Proc Natl Acad Sci U S A 2011;108(22):9232-9237

78 Gerin I, Clerbaux LA, Haumont O, et al. Expression of miR-33 from an SREBP2 intron inhibits cholesterol export and fatty acid oxidation. J Biol Chem 2010;285(44):33652-33661

79 Marquart TJ, Allen RM, Ory DS, Baldán A. miR-33 links SREBP-2 induction to repression of sterol transporters. Proc Natl Acad Sci U S A 2010;107(27):12228-12232

80 Najafi-Shoushtari SH, Kristo F, Li Y, et al. MicroRNA-33 and the SREBP host genes cooperate to control cholesterol homeostasis. Science 2010;328(5985):1566-1569

81 Rayner KJ, Suárez Y, Dávalos A, et al. MiR-33 contributes to the regulation of cholesterol homeostasis. Science 2010;328(5985): 1570-1573

82 Horie T, Ono K, Horiguchi M, et al. MicroRNA-33 encoded by an intron of sterol regulatory element-binding protein 2 (Srebp2) regulates HDL in vivo. Proc Natl Acad Sci U S A 2010;107(40): 17321-17326

83 Näär AM. Anti-atherosclerosis or no anti-atherosclerosis: that is the miR-33 question. Arterioscler Thromb Vasc Biol 2013;33(3): 447-448

84 Rayner KJ, Sheedy FJ, Esau CC, et al. Antagonism of miR-33 in mice promotes reverse cholesterol transport and regression of atherosclerosis. J Clin Invest 2011;121(7):2921-2931

85 Horie T, Baba O, Kuwabara Y, et al. MicroRNA-33 deficiency reduces the progression of atherosclerotic plaque in ApoE-/mice. J Am Heart Assoc 2012;1(6):e003376

86 Rayner KJ, Esau CC, Hussain FN, et al. Inhibition of miR-33a/b in non-human primates raises plasma HDL and lowers VLDL triglycerides. Nature 2011;478(7369):404-407

87 Marquart TJ, Wu J, Lusis AJ, Baldán A. Anti-miR-33 therapy does not alter the progression of atherosclerosis in low-density lipoprotein receptor-deficient mice. Arterioscler Thromb Vasc Biol 2013;33(3):455-458

88 Rotllan N, Ramírez CM, Aryal B, Esau CC, Fernández-Hernando C. Therapeutic silencing of microRNA-33 inhibits the progression of atherosclerosis in Ldlr-/- Mice-brief report. Arterioscler Thromb Vasc Biol 2013;33(8):1973-1977

89 Jeon TI, Esquejo RM, Roqueta-Rivera M, et al. An SREBP-responsive microRNA operon contributes to a regulatory loop for intracellular lipid homeostasis. Cell Metab 2013;18(1):51-61

90 Rufo C, Teran-Garcia M, Nakamura MT, Koo SH, Towle HC, Clarke $\mathrm{SD}$. Involvement of a unique carbohydrate-responsive factor in the glucose regulation of rat liver fatty-acid synthase gene transcription. J Biol Chem 2001;276(24):21969-21975

91 Shih HM, Liu Z, Towle HC. Two CACGTG motifs with proper spacing dictate the carbohydrate regulation of hepatic gene transcription. J Biol Chem 1995;270(37):21991-21997
92 Ma L, Tsatsos NG, Towle HC. Direct role of ChREBP.Mlx in regulating hepatic glucose-responsive genes. J Biol Chem 2005; 280(12):12019-12027

93 Stoeckman AK, Ma L, Towle HC. Mlx is the functional heteromeric partner of the carbohydrate response element-binding protein in glucose regulation of lipogenic enzyme genes. J Biol Chem 2004; 279(15):15662-15669

94 Sakiyama H, Wynn RM, Lee WR, et al. Regulation of nuclear import/export of carbohydrate response element-binding protein (ChREBP): interaction of an alpha-helix of ChREBP with the 14-3-3 proteins and regulation by phosphorylation. J Biol Chem 2008;283(36):24899-24908

95 Kawaguchi T, Osatomi K, Yamashita H, Kabashima T, Uyeda K. Mechanism for fatty acid "sparing" effect on glucose-induced transcription: regulation of carbohydrate-responsive elementbinding protein by AMP-activated protein kinase. J Biol Chem 2002;277(6):3829-3835

96 Kabashima T, Kawaguchi T, Wadzinski BE, Uyeda K. Xylulose 5phosphate mediates glucose-induced lipogenesis by xylulose 5phosphate-activated protein phosphatase in rat liver. Proc Natl Acad Sci U S A 2003;100(9):5107-5112

97 Tsatsos NG, Towle HC. Glucose activation of ChREBP in hepatocytes occurs via a two-step mechanism. Biochem Biophys Res Commun 2006;340(2):449-456

98 Bricambert J, Miranda J, Benhamed F, Girard J, Postic C, Dentin R. Salt-inducible kinase 2 links transcriptional coactivator p300 phosphorylation to the prevention of ChREBP-dependent hepatic steatosis in mice. J Clin Invest 2010;120(12):4316-4331

99 Guinez C, Filhoulaud G, Rayah-Benhamed F, et al. O-GlcNAcylation increases ChREBP protein content and transcriptional activity in the liver. Diabetes 2011;60(5):1399-1413

100 Sakiyama H, Fujiwara N, Noguchi T, et al. The role of O-linked GlcNAc modification on the glucose response of ChREBP. Biochem Biophys Res Commun 2010;402(4):784-789

101 Davies MN, O'Callaghan BL, Towle HC. Activation and repression of glucose-stimulated ChREBP requires the concerted action of multiple domains within the MondoA conserved region. Am J Physiol Endocrinol Metab 2010;299(4):E665-E674

102 Li MV, Chang B, Imamura M, Poungvarin N, Chan L. Glucosedependent transcriptional regulation by an evolutionarily conserved glucose-sensing module. Diabetes 2006;55(5):1179-1189

103 Li MV, Chen W, Poungvarin N, Imamura M, Chan L. Glucosemediated transactivation of carbohydrate response elementbinding protein requires cooperative actions from Mondo conserved regions and essential trans-acting factor 14-3-3. Mol Endocrinol 2008;22(7):1658-1672

104 McFerrin LG, Atchley WR. A novel N-terminal domain may dictate the glucose response of Mondo proteins. PLoS ONE 2012;7(4): e34803

105 Dentin R, Tomas-Cobos L, Foufelle F, et al. Glucose 6-phosphate, rather than xylulose 5-phosphate, is required for the activation of ChREBP in response to glucose in the liver. J Hepatol 2012;56(1): 199-209

106 Iizuka K, Takeda J, Horikawa Y. Hepatic overexpression of dominant negative Mlx improves metabolic profile in diabetes-prone C57BL/6J mice. Biochem Biophys Res Commun 2009;379(2): 499-504

107 Iizuka K, Wu W, Horikawa Y, Takeda J. Role of glucose-6-phosphate and xylulose-5-phosphate in the regulation of glucosestimulated gene expression in the pancreatic $\beta$ cell line, INS-1E. Endocr J 2013;60(4):473-482

108 Herman MA, Peroni OD, Villoria J, et al. A novel ChREBP isoform in adipose tissue regulates systemic glucose metabolism. Nature 2012;484(7394):333-338

109 Dentin R, Benhamed F, Hainault I, et al. Liver-specific inhibition of ChREBP improves hepatic steatosis and insulin resistance in ob/ ob mice. Diabetes 2006;55(8):2159-2170 
110 Iizuka K, Miller B, Uyeda K. Deficiency of carbohydrate-activated transcription factor ChREBP prevents obesity and improves plasma glucose control in leptin-deficient (ob/ob) mice. Am J Physiol Endocrinol Metab 2006;291(2):E358-E364

111 Benhamed F, Denechaud PD, Lemoine M, et al. The lipogenic transcription factor ChREBP dissociates hepatic steatosis from insulin resistance in mice and humans. J Clin Invest 2012;122(6): 2176-2194

112 He Z, Jiang T, Wang Z, Levi M, Li J. Modulation of carbohydrate response element-binding protein gene expression in 3T3-L1 adipocytes and rat adipose tissue. Am J Physiol Endocrinol Metab 2004;287(3):E424-E430

113 Wang H, Kouri G, Wollheim CB. ER stress and SREBP-1 activation are implicated in beta-cell glucolipotoxicity. J Cell Sci 2005;118(Pt 17):3905-3915

114 Metukuri MR, Zhang P, Basantani MK, et al. ChREBP mediates glucose-stimulated pancreatic $\beta$-cell proliferation. Diabetes 2012;61(8):2004-2015

115 Brown MS, Goldstein JL. Selective versus total insulin resistance: a pathogenic paradox. Cell Metab 2008;7(2):95-96 